Thermal Science and Engineering (2019) Volume 2 doi:10.24294/tse.v2i2.181

\title{
Anisotropic Behaviour and Elastic Moduli of Average Isotropic and Anisotropic Elastic Media
}

\author{
Tleukenov S. ${ }^{*}$ Bobeev A., Sabitova D.
}

L.N.Gumilyov Eurasian National University, Astana, Republic of Kazakhstan, 010008

\section{ABSTRACT}

Using matricant method elastic moduli of occasionally heterogeneous isotropic and anisotropic elastic media were received. Anisotropic behaviour and conditions for change in anisotropy of media associated with averaging of one-dimensional periodic structures was determined.

PACS Numbers: PACS: 43.20.Bi

\section{Introduction}

Inhomogeneity and anisotropy is the most widespread behaviour of real media. When studying wave process in Earth crust and in composite mechanics a model of homogeneous or layered media with average characteristics is widely used as a real media model. Currently because of intensive development and application of non-destructive methods, sound velocity methods, tomography, remote sensing, etc. similar models are used in various fields of sci- ence. In case of assumption of heterogeneous medium with homogeneous model application of the most adequate model of primary medium is important. This work sets the results of investigation of anisotropic behaviour of homogeneous models of heterogeneous isotropic and anisotropic elastic media. Based on matricant method ${ }^{[1-3]}$ the formulae determining values of elastic media were received. Research method determines anisotropy of averaged media.

\section{Elastic models of averaged occasionally isotropic medium}

Within the frames of matricant method and equivalent system of ordinary differen- tial first-order equations describing propagation of elastic harmonic wave in heterogeneous isotropic medium in case of inhomogeneity along axis $\mathrm{z}$ is written as:

$\frac{d \vec{W}}{d z}=B(z) \vec{W} ; \vec{W}=\left(U_{z}, \sigma_{z z}, U_{x}, \sigma_{x z}, U_{y}, \sigma_{y z}\right)^{t}$.
Matrix of coefficients $B(z)$ is structured as:

$$
B=\left(\begin{array}{cccccc}
0 & b_{12} & b_{13} & 0 & b_{15} & 0 \\
b_{21} & 0 & 0 & b_{24} & 0 & b_{26} \\
b_{24} & 0 & 0 & b_{34} & 0 & 0 \\
0 & b_{13} & b_{43} & 0 & b_{45} & 0 \\
b_{26} & 0 & 0 & 0 & 0 & b_{56} \\
0 & b_{15} & b_{45} & 0 & b_{65} & 0
\end{array}\right)
$$

Elements $b_{i j}(\mathrm{z})$ :

$$
\begin{aligned}
& b_{11}=\frac{1}{c_{11}} ; b_{13}=i k_{x} \frac{c_{12}}{c_{11}} ; b_{15}=i k_{y} \frac{c_{12}}{c_{11}} ; \\
& b_{21}=-\omega^{2} \rho ; b_{24}=i k_{x} ; b_{26}=i k_{y} ; \\
& b_{34}=\frac{1}{c_{66}} ; b_{43}=-\omega^{2} \rho+k_{x}^{2}\left(c_{11}-\frac{c_{12}^{2}}{c_{11}}\right)+k_{y}^{2} c_{66} ;
\end{aligned}
$$

$$
b_{45}=k_{x} k_{y}\left(c_{66}+c_{12}-\frac{c_{12}^{2}}{c_{11}}\right) ; b_{56}=\frac{1}{c_{66}}
$$$$
b_{65}=-\omega^{2} \rho+k_{x}^{2} c_{66}+k_{y}^{2}\left(c_{11}-\frac{c_{12}^{2}}{c_{11}}\right) \text {. }
$$

In case of isotropic media:

$$
\begin{aligned}
& {\left[c_{11}=c_{22}=c_{33}=\lambda+2 \mu=c_{11} ;\right.} \\
& {\left[c_{11}=c_{22}=c_{33}=\lambda+2 \mu=c_{11} ;\right.} \\
& c_{44}=c_{55}=c_{66}=\mu=\frac{1}{2}\left(c_{11}-c_{12}\right)=c_{66} .
\end{aligned}
$$

Given periodic inhomogeneity of medium:

$$
\begin{aligned}
& \lambda(z)=\lambda(z+h) ; \mu(z)=\mu(z+h) ; \\
& c_{i j}(z)=c_{i j}\left(\lambda_{0}+h\right),
\end{aligned}
$$


where $h$ is inhomogeneity period.

If wave length is $\lambda_{0} \gg h$, occasionally heterogeneous medium can be considered as homogeneous medium described by matrix of coefficients as averaged based on inhomogeneity period:

$$
\frac{d \vec{W}}{d z}=\langle B\rangle \vec{W} ;\langle B\rangle=\frac{1}{h} \int_{0}^{h} B(z) d z .
$$

From (6) it follows:

$$
\begin{gathered}
\left\langle b_{i j}\right\rangle=\frac{1}{h} \int_{0}^{h} b_{i j}(z) d z ; \\
\left\langle b_{11}\right\rangle=\left\langle\frac{1}{c_{11}}\right\rangle ;\left\langle b_{13}\right\rangle=i k_{x}\left\langle\frac{c_{12}}{c_{11}}\right\rangle \\
\left\langle b_{15}\right\rangle=i k_{y}\left\langle\frac{c_{12}}{c_{11}}\right\rangle ;\left\langle b_{34}\right\rangle=\left\langle\frac{1}{c_{66}}\right\rangle ; \\
\left\langle b_{21}\right\rangle=-\omega^{2}\langle\rho\rangle ;\left\langle b_{24}\right\rangle=i k_{x} ;\left\langle b_{26}\right\rangle=i k_{y} ; \\
\left\langle b_{43}\right\rangle=-\omega^{2}\langle\rho\rangle+k_{x}^{2}\left\langle c_{11}-\frac{c_{12}^{2}}{c_{11}}\right\rangle+k_{y}^{2}\left\langle c_{66}\right\rangle ; \\
\left\langle b_{45}\right\rangle=k_{x} k_{y}\left\langle c_{66}+c_{12}-\frac{c_{12}^{2}}{c_{11}}\right\rangle ;\left\langle b_{56}\right\rangle=\left\langle\frac{1}{c_{66}}\right\rangle ; \\
\left\langle b_{65}\right\rangle=-\omega^{2}\langle\rho\rangle+k_{x}^{2}\left\langle c_{66}\right\rangle+k_{y}^{2}\left\langle c_{11}-\frac{c_{12}^{2}}{c_{11}}\right\rangle .
\end{gathered}
$$

Similar system of first-order equations for homogeneous elastic medium of hexagonal anisotropy (axis z $\| A_{6}$ is hexagonal axis) is written as:

$\frac{d \vec{W}}{d z}=B \vec{W} ; \vec{W}=\left(U_{z}, \sigma_{z z}, U_{x}, \sigma_{x z}, U_{y}, \sigma_{y z}\right)^{t}$

Structure of matrix of coefficients $B$ in (8) is the same as (2). Elements $b_{i j}$ are written as:

$$
b_{11}=\frac{1}{c_{22}^{0}} ; b_{12}=i k_{x} \frac{c_{13}^{0}}{c_{33}^{0}} ; b_{15}=i k_{y} \frac{c_{13}^{0}}{c_{33}^{0}} ;
$$

$b_{21}=-\omega^{2} \rho ; b_{24}=i k_{x} ; b_{26}=i k_{y}$;

$b_{34}=\frac{1}{c_{44}^{0}} ; b_{43}=-\omega^{2} \rho+k_{x}^{2}\left(c_{11}^{0}-\frac{c_{13}^{0}{ }^{2}}{c_{33}^{0}}\right)+k_{y}^{2} c_{66}^{0}$;

$b_{45}=k_{x} k_{y}\left(c_{66}^{0}+c_{12}^{0}-\frac{c_{13}^{0}{ }^{2}}{c_{33}^{0}}\right)$

$b_{56}=\frac{1}{c_{44}^{0}} ; b_{65}=-\omega^{2} \rho_{0}+k_{x}^{2} c_{66}^{0}+k_{y}^{2}\left(c_{11}^{0}-\frac{{c_{13}^{0}}^{2}}{c_{33}^{0}}\right)$

Comparison of elements of averaged matrix (7) and matrix of coefficients of homogeneous anisotropic medium of hexagonal symmetry (9) ends in relations:

$$
\left\langle\frac{1}{c_{11}}\right\rangle=\frac{1}{c_{33}^{0}} ;\left\langle\frac{1}{c_{66}}\right\rangle=\frac{1}{c_{44}^{0}} ;\left\langle c_{66}\right\rangle=c_{66}^{0} ;
$$

$$
\begin{aligned}
& \langle\rho\rangle=\rho_{0} ;\left\langle\frac{c_{12}}{c_{11}}\right\rangle=\frac{c_{13}^{0}}{c_{33}^{0}} ;\left\langle c_{11}-\frac{c_{12}^{2}}{c_{33}}\right\rangle=c_{11}^{0}-\frac{c_{13}^{0}{ }^{2}}{c_{33}^{0}} ; \\
& \left\langle c_{12}-\frac{c_{12}^{2}}{c_{11}}\right\rangle=c_{12}^{0}-\frac{c_{13}^{0}}{c_{33}^{0}} .
\end{aligned}
$$

From (10) it follows:

$$
\begin{aligned}
& c_{33}^{0}=\left\langle\frac{1}{c_{11}}\right\rangle^{-1} ; c_{44}^{0}=\left\langle\frac{1}{c_{66}}\right\rangle^{-1} ; c_{66}^{0}=\left\langle c_{66}\right\rangle ; \\
& c_{13}^{0}=\left\langle\frac{c_{12}}{c_{11}}\right\rangle\left\langle\frac{1}{c_{11}}\right\rangle^{-1}=\left\langle\frac{c_{12}}{c_{11}}\right\rangle c_{33}^{0} ; \\
& c_{11}^{0}=\left\langle c_{11}\right\rangle-\left\langle\frac{c_{12}^{2}}{c_{11}}\right\rangle+\frac{c_{13}^{0}}{c_{33}^{0}} \\
& c_{12}^{0}=\left\langle c_{12}\right\rangle-\left\langle\frac{c_{12}^{2}}{c_{11}}\right\rangle+\frac{c_{13}^{0}}{c_{33}^{0}} ; c_{66}^{0}=\frac{1}{2}\left(c_{11}^{0}-c_{12}^{0}\right)
\end{aligned}
$$

Elastic modules $\mathrm{c}_{i i}^{0}$ match up with elastic modules of hexagonal anisotropy.

Thus, an averaged heterogeneous isotropic medium is equivalent to homogeneous elastic medium of hexagonal anisotropy with elastic modules:

$$
\begin{gathered}
c_{33}^{0}=\left\langle\frac{1}{c_{11}}\right\rangle^{-1}=\left\langle\frac{1}{\lambda+2 \mu}\right\rangle^{-1} ; c_{44}^{0}=\left\langle\frac{1}{c_{44}}\right\rangle^{-1}=\left\langle\frac{1}{\mu}\right\rangle^{-1} ; \\
\langle\rho\rangle=\rho_{0} ; c_{66}^{0}=\frac{1}{2}\left(c_{11}^{0}-c_{12}^{0}\right)=\langle\mu\rangle ;
\end{gathered}
$$

$$
\begin{gathered}
c_{11}^{0}=\langle\lambda+2 \mu\rangle-\left\langle\frac{\lambda^{2}}{\lambda+2 \mu}\right\rangle+\left\langle\frac{\lambda}{\lambda+2 \mu}\right\rangle^{2}\left\langle\frac{\lambda}{\lambda+2 \mu}\right\rangle_{(12}^{-1} ; \\
c_{12}^{0}=\langle\lambda\rangle-\left\langle\frac{\lambda^{2}}{\lambda+2 \mu}\right\rangle+\left\langle\frac{\lambda}{\lambda+2 \mu}\right\rangle^{2}\left\langle\frac{\lambda}{\lambda+2 \mu}\right\rangle^{-1} ; \\
c_{13}^{0}=\left\langle\frac{\lambda}{\lambda+2 \mu}\right\rangle\left\langle\frac{\lambda}{\lambda+2 \mu}\right\rangle^{-1} . \\
c_{66}^{0}=\frac{1}{2}\left(c_{11}^{0}-c_{12}^{0}\right)=\frac{1}{2}(\langle\lambda+2 \mu\rangle-\langle\lambda\rangle)=\langle\mu\rangle .
\end{gathered}
$$

Averaging of elastic medium of hexagonal anisotropy occasionally heterogeneous along axis $\mathrm{z}(\mathrm{z} \|$ $A_{6}$ ) leaves anisotropic behaviour unaffected.

From averaging of matrix of coefficients $B$ (9) under its occasional dependence:

$$
b_{i j}(z)=b_{i j}(z+h)
$$

it follows:

$$
\begin{aligned}
& c_{33}^{0}=\left\langle\frac{1}{c_{33}}\right\rangle ; c_{44}^{0}=\left\langle\frac{1}{c_{44}}\right\rangle^{-1} ; \\
& c_{66}^{0}=\left\langle c_{66}\right\rangle\langle\rho\rangle=\rho_{0} ; c_{13}^{0}=\left\langle\frac{c_{13}}{c_{33}}\right\rangle\left\langle\frac{1}{c_{33}}\right\rangle^{-1} ;
\end{aligned}
$$




$$
\begin{aligned}
& c_{12}^{0}=\left\langle c_{12}\right\rangle-\left\langle\frac{c_{13}^{2}}{c_{33}}\right\rangle+\frac{c_{13}^{0}{ }^{2}}{c_{33}} ; c_{11}^{0}=\left\langle c_{11}\right\rangle-\left\langle\frac{c_{13}^{2}}{c_{33}}\right\rangle+\frac{c_{13}^{02}}{c_{33}} ; \\
& c_{66}^{0}=\left\langle c_{66}\right\rangle=\frac{1}{2}\left\langle c_{11}\right\rangle-\frac{1}{2}\left\langle c_{12}\right\rangle
\end{aligned}
$$

From now on $c_{i j}$ is elastic models of heterogeneous medium; $c_{i i}^{0}$ - elastic models of homogeneous averaged medium.

Conclusion: inhomogeneity of anisotropic medium of hexagonal symmetry along axiz $z$, parallel hexagonal axis $\mathrm{A}_{6}$, leaves its anisotropic behaviour unaffected.

Averaged heterogeneous anisotropic medium of hexagonal symmetry along hexagonal axis has anisotropic behaviour of hexagonal symmetry.

Anisotropic behaviour remains unchanged.

\section{Elastic}

modules

\section{anisotropic}

behaviour

and occasionally heterogeneous media of hexagonal symmetries averaged along axis $\mathrm{x} \perp \mathbf{A}_{6}$

Within the frames of matricant method wave process in anisotropic media of hexagonal symmetry heterogeneous along axis $\mathrm{x}$ are described by the system of ordinary differential first-order equations:

$$
\frac{d \vec{W}}{d x}=B(x) \vec{W} ; \vec{W}=\left(U_{x}, \sigma_{x x}, U_{y}, \sigma_{y x}, U_{z}, \sigma_{x z}\right)^{t} .
$$

Matrix of coefficients $B(\mathrm{x})$ is structured as (2) with elements $b_{i j}(\mathrm{x})$ :

$$
\begin{aligned}
& b_{12}=\frac{1}{c_{11}} ; b_{13}=i k_{y} \frac{c_{12}}{c_{11}} b_{15}=i k_{z} \frac{c_{13}}{c_{11}} ; \\
& b_{21}=-\omega^{2} \rho ; b_{24}=i k_{y} ; b_{26}=i k_{z} ; b_{34}=\frac{1}{c_{66}} ; \\
& b_{43}=-\omega^{2} \rho+k_{z}^{2} c_{44}+k_{y}^{2}\left(c_{11}-\frac{c_{12}^{2}}{c_{11}}\right) ; \\
& b_{45}=k_{y} k_{z}\left(c_{44}+c_{13}-\frac{c_{12} c_{13}}{c_{11}}\right) ; \\
& b_{56}=\frac{1}{c_{44}} ; b_{65}=-\omega^{2} \rho+k_{y}^{2} c_{44}+k_{z}^{2}\left(c_{33}-\frac{c_{13}^{2}}{c_{11}}\right)
\end{aligned}
$$

In case of occasional inhomogeneity along $\mathrm{x}$ :

$$
\rho(x)=\rho(x+h) ; ; c_{i j}(x)=c_{i j}(x+h) .
$$

Assuming that $\lambda_{0} \gg \mathrm{h}\left(\lambda_{0}\right.$ is wave length, $\mathrm{h}$ is inhomogeneity period) from

$$
\langle B(x)\rangle=\frac{1}{h} \int_{0}^{h} B(x) d x
$$

elements of averaged matrix of coefficient follow:

$$
\begin{aligned}
& \left\langle b_{12}\right\rangle=\left\langle\frac{1}{c_{11}}\right\rangle ; b_{13}=i k_{y}\left\langle\frac{c_{12}}{c_{11}}\right\rangle ; b_{15}=i k_{z}\left\langle\frac{c_{13}}{c_{11}}\right\rangle ; \\
& \left\langle b_{21}\right\rangle=-\omega^{2}\langle\rho\rangle ;\left\langle b_{34}\right\rangle=\left\langle\frac{1}{c_{66}}\right\rangle ; \\
& \left\langle b_{43}\right\rangle=-\omega^{2}\langle\rho\rangle+k_{y}^{2}\left\langle c_{11}-\frac{c_{12}^{2}}{c_{11}}\right\rangle+k_{z}^{2}\left\langle c_{44}\right\rangle ; \\
& \left\langle b_{45}\right\rangle=k_{y} k_{z}\left\langle c_{44}+c_{13}-\frac{c_{12} c_{13}}{c_{11}}\right\rangle ; \\
& \left\langle b_{56}\right\rangle=\left\langle\frac{1}{c_{44}}\right\rangle ;\left\langle b_{65}\right\rangle=-\omega^{2}\langle\rho\rangle+k_{y}^{2}\left\langle c_{44}\right\rangle+k_{z}^{2}\left\langle c_{33}-\frac{c_{13}^{2}}{c_{11}}\right\rangle
\end{aligned}
$$

Set of equations for anisotropic media of rhombic symmetry is written as (15) with the structure of matrix of coefficients $B$ as (2).

Elements of matrix $\mathrm{B}$ for media of rhombic symmetry are written as:

$$
\begin{aligned}
& b_{12}=\frac{1}{c_{11}^{0}} ; b_{13}=i k_{y} \frac{c_{12}^{0}}{c_{11}^{0}} ; b_{15}=i k_{z} \frac{c_{13}^{0}}{c_{11}^{0}} ; b_{21}=-\omega^{2} \rho_{0} ; \\
& b_{24}=i k_{y} ; b_{26}=i k_{z} ; b_{34}=\frac{1}{c_{66}^{0}} ; \\
& b_{43}=-\omega^{2} \rho_{0}+k_{z}^{2} c_{44}^{0}+k_{y}^{2}\left(c_{22}-\frac{c_{12}^{0}}{c_{11}^{0}}\right) ; \\
& b_{45}=k_{y} k_{z}\left(c_{44}^{0}+c_{23}^{0}-\frac{c_{12}^{0} c_{13}^{0}}{c_{11}^{0}}\right) ; \\
& b_{56}=\frac{1}{c_{55}^{0}} ; b_{65}=-\omega^{2} \rho_{0}+k_{y}^{2} c_{44}^{0}+k_{z}^{2}\left(c_{33}^{0}-\frac{c_{13}^{0}{ }^{2}}{c_{11}^{0}}\right)
\end{aligned}
$$

From comparison of bij (19) and (20) we obtain:

$$
\begin{gathered}
\left\langle\frac{1}{c_{11}}\right\rangle=\frac{1}{c_{11}^{0}} ;\left\langle\frac{c_{12}}{c_{11}}\right\rangle=\frac{c_{12}^{0}}{c_{11}^{0}} ;\left\langle\frac{c_{13}}{c_{11}}\right\rangle=\frac{c_{13}^{0}}{c_{11}^{0}} ; \\
\langle\rho\rangle=\rho_{0} ;\left\langle c_{44}\right\rangle=c_{44}^{0} ;\left\langle c_{11}-\frac{c_{12}^{2}}{c_{11}}\right\rangle=c_{22}^{0}-\frac{c_{12}^{0}}{c_{11}^{0}} ; \\
\left\langle c_{13}-\frac{c_{12} c_{13}}{c_{11}}\right\rangle=c_{23}^{0}-\frac{c_{12}^{0} c_{13}^{0}}{c_{11}^{0}} ;\left\langle\frac{1}{c_{44}}\right\rangle=\frac{1}{c_{55}^{0}} .
\end{gathered}
$$

From (21) it follows:

$$
c_{11}^{0}=\left\langle\frac{1}{c_{11}}\right\rangle^{-1} ; c_{12}^{0}=\left\langle\frac{c_{12}}{c_{11}}\right\rangle c_{11}^{0} ;
$$




$$
\begin{aligned}
& c_{13}^{0}=\left\langle\frac{c_{13}}{c_{11}}\right\rangle c_{11}^{0} ; c_{44}^{0}=\left\langle c_{44}\right\rangle ; \\
& c_{55}^{0}=\left\langle\frac{1}{c_{44}}\right\rangle^{-1} ; c_{66}^{0}=\left\langle\frac{1}{c_{66}}\right\rangle^{-1} ; \\
& c_{22}^{0}=\left\langle c_{11}\right\rangle-\left\langle\frac{c_{12}^{2}}{c_{11}}\right\rangle+\frac{c_{12}^{0}}{c_{11}^{0}} ; \\
& c_{23}^{0}=\left\langle c_{13}\right\rangle-\left\langle\frac{c_{12} c_{13}}{c_{11}}\right\rangle+\frac{c_{12}^{0} c_{13}^{0}}{c_{11}} ; \\
& c_{33}^{0}=\left\langle c_{33}\right\rangle-\left\langle\frac{c_{13}^{2}}{c_{11}}\right\rangle+\frac{c_{13}^{0}}{c_{11}^{0}} .
\end{aligned}
$$

We obtained nine independent elastic modules derived from averaging of matrix of coefficients $B(x)$ along $x$ :

$$
c_{11}^{0}, c_{22}^{0}, c_{33}^{0}, c_{44}^{0}, c_{55}^{0}, c_{66}^{0} ; c_{12}^{0}, c_{13}^{0}, c_{23}^{0} .
$$

\section{Elastic \\ modules \\ anisotropic behaviour \\ and occasionally heterogeneous media of hexagonal symmetry averaged along axis $\mathbf{y} \perp \mathbf{A}_{6}$}

First-order system is written as:

$$
\frac{d \vec{W}}{d y}=B(y) \vec{W} ; \vec{W}=\left(U_{y}, \sigma_{y y}, U_{x}, \sigma_{x y}, U_{z}, \sigma_{z y}\right)^{t} .
$$

Structure of matrix of coefficients $B(y)$ in (24) is the same as (2). Elements $b_{i j}$ of averaged matrix $B(y)$

$$
\langle B\rangle=\frac{1}{h} \int_{0}^{h} B(y) d y
$$

by inhomogeneity period are written as:

$$
\begin{aligned}
& \left\langle b_{12}\right\rangle=\left\langle\frac{1}{c_{11}}\right\rangle ; b_{13}=i k_{x}\left\langle\frac{c_{12}}{c_{11}}\right\rangle ; \\
& b_{15}=i k_{z}\left\langle\frac{c_{13}}{c_{11}}\right\rangle ;\left\langle b_{21}\right\rangle=-\omega^{2}\langle\rho\rangle ; \\
& \left\langle b_{34}\right\rangle=\left\langle\frac{1}{c_{66}}\right\rangle ;\left\langle b_{43}\right\rangle=-\omega^{2}\langle\rho\rangle+k_{x}^{2}\left\langle c_{11}-\frac{c_{12}^{2}}{c_{11}}\right\rangle+k_{z}^{2}\left\langle c_{44}\right\rangle ; \\
& \left\langle b_{45}\right\rangle=k_{x} k_{z}\left\langle c_{44}+c_{13}-\frac{c_{12} c_{13}}{c_{11}}\right\rangle ;\left\langle b_{56}\right\rangle=\left\langle\frac{1}{c_{44}}\right\rangle ; \\
& \left\langle b_{65}\right\rangle=-\omega^{2}\langle\rho\rangle+k_{x}^{2}\left\langle c_{44}\right\rangle+k_{z}^{2}\left\langle c_{33}-\frac{c_{13}^{2}}{c_{11}}\right\rangle .
\end{aligned}
$$

Set of equations and vector $\underset{w}{\rightarrow}$ for anisotropic elastic medium of rhombic symmetry are written as (24).

Structure of B is also written as (2).
Elements $b_{i j}$ for media of rhombic anisotropy:

$$
\begin{aligned}
& b_{12}=\frac{1}{c_{22}^{0}} ; b_{13}=i k_{x} \frac{c_{12}^{0}}{c_{22}^{0}} ; b_{15}=i k_{z} \frac{c_{23}^{0}}{c_{22}^{0}} \\
& b_{21}=-\omega^{2} \rho_{0} ; b_{24}=i k_{x} ; b_{26}=i k_{z} ; \\
& b_{34}=\frac{1}{c_{66}^{0}} ; b_{43}=-\omega^{2} \rho_{0}+k_{x}^{2}\left(c_{11}^{0}-\frac{c_{12}^{0}}{c_{22}^{0}}\right)+k_{z}^{2} c_{55}^{0} ; \\
& b_{45}=k_{x} k_{z}\left(c_{55}^{0}+c_{13}^{0}-\frac{c_{12}^{0} c_{23}^{0}}{c_{22}^{0}}\right) ; b_{56}=\frac{1}{c_{44}^{0}} \\
& b_{65}=-\omega^{2} \rho_{0}+k_{x}^{2} c_{55}^{0}+k_{z}^{2}\left(c_{33}^{0}-\frac{c_{23}^{0}}{c_{22}^{0}}\right)
\end{aligned}
$$

Likewise, comparison of (26) and (27) determine elastic modules of averaged medium.

$$
\begin{aligned}
& c_{22}^{0}=\left\langle\frac{1}{c_{11}}\right\rangle^{-1} ; c_{12}^{0}=\left\langle\frac{c_{12}}{c_{11}}\right\rangle\left\langle\frac{1}{c_{11}}\right\rangle^{-1}=\left\langle\frac{c_{12}}{c_{11}}\right\rangle c_{22}^{0} ; \\
& c_{13}^{0}=\left\langle\frac{c_{13}}{c_{11}}\right\rangle c_{22}^{0} ; c_{66}^{0}=\left\langle\frac{1}{c_{66}}\right\rangle^{-1} ; \\
& c_{55}^{0}=\left\langle c_{44}\right\rangle ; c_{44}^{0}=\left\langle\frac{1}{c_{44}}\right\rangle^{-1} ; c_{11}^{0}=\left\langle c_{11}\right\rangle-\left\langle\frac{c_{12}^{2}}{c_{11}}\right\rangle+\frac{c_{12}^{0}}{c_{22}^{0}} ; \\
& c_{13}^{0}=\left\langle c_{13}\right\rangle-\left\langle\frac{c_{12} c_{13}}{c_{11}}\right\rangle+\frac{c_{12}^{0} c_{13}^{0}}{c_{22}^{0}} ; c_{33}^{0}=\left\langle c_{33}\right\rangle-\left\langle\frac{c_{13}^{2}}{c_{11}}\right\rangle+\frac{c_{23}^{0}}{c_{22}^{0}} .
\end{aligned}
$$

Nine independent elastic modules:

$$
c_{11}^{0}, c_{22}^{0}, c_{33}^{0}, c_{44}^{0}, c_{55}^{0}, c_{66}^{0} ; c_{12}^{0}, c_{13}^{0}, c_{23}^{0} \text {. }
$$

Also determine rhombic anisotropy of averaged elastic anisotropic medium of hexagonal symmetry occasionally heterogeneous along axis $y$.

\section{Anisotropic media of rhombic symmetry}

Averaging of occasionally heterogeneous anisotropic medium of rhombic symmetry along axis $x$ ends in:

$$
\begin{aligned}
\langle B\rangle & =\frac{1}{h} \int_{0}^{h} B(x) d x ; \\
\left\langle b_{12}\right\rangle & =\left\langle\frac{1}{c_{11}}\right\rangle ; b_{13}=i k_{y}\left\langle\frac{c_{12}}{c_{11}}\right\rangle ; b_{15}=i k_{z}\left\langle\frac{c_{13}}{c_{11}}\right\rangle ; \\
\left\langle b_{21}\right\rangle & =-\omega^{2}\langle\rho\rangle ;\left\langle b_{34}\right\rangle=\left\langle\frac{1}{c_{66}}\right\rangle ; \\
\left\langle b_{43}\right\rangle & =-\omega^{2}\langle\rho\rangle+k_{z}^{2}\left\langle c_{44}\right\rangle+\left\langle c_{22}-\frac{c_{12}^{2}}{c_{11}}\right\rangle k_{y}^{2} ; \\
\left\langle b_{56}\right\rangle & =\left\langle\frac{1}{c_{55}}\right\rangle ; \\
\left\langle b_{45}\right\rangle & =k_{y} k_{z}\left\langle c_{44}+c_{25}-\frac{c_{12} c_{13}}{c_{11}}\right\rangle
\end{aligned}
$$




$$
\left\langle b_{65}\right\rangle=-\omega^{2}\langle\rho\rangle+\left\langle c_{44}\right\rangle k_{y}^{2}+\left\langle c_{33}-\frac{c_{13}^{2}}{c_{11}}\right\rangle k_{z}^{2} .
$$

Comparison with elements $b_{i j}$ using rhombically anisotropic medium gives:

$$
\begin{aligned}
& \left\langle\frac{1}{c_{11}}\right\rangle=\frac{1}{c_{11}^{0}} ;\left\langle\frac{c_{12}}{c_{11}}\right\rangle=\frac{c_{12}^{0}}{c_{11}^{0}} ;\left\langle\frac{c_{13}}{c_{11}}\right\rangle=\frac{c_{13}^{0}}{c_{11}^{0}} ; \\
& \langle\rho\rangle=\rho_{0} ;\left\langle\frac{1}{c_{66}}\right\rangle=\frac{1}{c_{66}^{0}} ;\left\langle c_{44}\right\rangle=c_{44}^{0} ; \\
& \left\langle c_{22}-\frac{c_{12}^{2}}{c_{11}}\right\rangle=c_{22}^{0}-\frac{c_{12}^{0}}{c_{11}^{0}} ;\left\langle c_{23}-\frac{c_{12} c_{13}}{c_{11}}\right\rangle=c_{23}^{0}-\frac{c_{12}^{0} c_{13}^{0}}{c_{11}^{0}} ; \\
& \left\langle\frac{1}{c_{55}}\right\rangle=\frac{1}{c_{55}^{0}} ;\left\langle c_{33}-\frac{c_{13}^{2}}{c_{11}}\right\rangle=c_{33}^{0}-\frac{c_{13}^{0}}{c_{11}^{0}} .
\end{aligned}
$$

It follows from these equations:

$$
\begin{aligned}
& c_{11}^{0}=\left\langle\frac{1}{c_{11}}\right\rangle^{-1} ; c_{44}^{0}=\left\langle c_{44}\right\rangle ; c_{55}^{0}=\left\langle\frac{1}{c_{55}}\right\rangle^{-1} ; c_{66}^{0}=\left\langle\frac{1}{c_{66}}\right\rangle^{-1} ; \\
& c_{12}^{0}=\left\langle\frac{c_{12}}{c_{11}}\right\rangle c_{11}^{0} ; c_{13}^{0}=\left\langle\frac{c_{13}}{c_{11}}\right\rangle c_{11}^{0} ; c_{22}^{0}=\left\langle c_{22}\right\rangle-\left\langle\frac{c_{12}^{2}}{c_{11}}\right\rangle+\frac{c_{12}^{0}}{c_{11}^{0}} ; \\
& c_{23}^{0}=\left\langle c_{23}\right\rangle-\left\langle\frac{c_{12} c_{13}}{c_{11}}\right\rangle+\frac{c_{12}^{0} c_{13}^{0}}{c_{11}^{0}} ; c_{33}^{0}=\left\langle c_{33}\right\rangle-\left\langle\frac{c_{13}^{2}}{c_{11}}\right\rangle+\frac{c_{13}^{0}}{c_{11}^{0}} .
\end{aligned}
$$

Elastic parameters of averaged medium (32) pertain to rhombic anisotropy again. Thus, further averaging of occasionally heterogeneous anisotropic media of rhombic symmetry along one of coordinate will not change anisotropic behaviour of media, only values, the variables of elastic modules change.

In case of averaging along axes $y$ or $z$ this conclusion is correct since anisotropic medium of rhombic symmetry has three orthogonal binary axes along which axes of reference are directed. These axes are equivalent.

\section{Conclusion}

Given typical scale of inhomogeneity of medium $\mathrm{h} \ll \lambda$ of wave length:

- elastic modules of averaging of one-dimensionally heterogeneous isotropic elastic media are determined

- elastic modules of averaging of one-dimensionally heterogeneous media of hexagonal anisotropy are determined:

a. along hexagonal axis

b. along axis orthogonal to hexagonal axis.

- elastic modules of averaging of one-dimensionally heterogeneous media of rhombic anisotropy along binary axis are determined.

From these redults it follows that:

- one-dimensionally heterogeneous isotropic media tend to hexagonal anisotropy;

- bi-dimesionally heterogeneous isotropic media tend to rhombic anisotropy;

- inhomogeneity along hexagonal axis in media of hexagonal aisotropy will not change medium symmetry;

- media of hexagonal anisotropy which are heterogenepus alon axes orthogonal to hexag- onal axis tend to media of rhombic anisotropy

- inhomogeneity along binary axes in rhombically anisotropic media will not change medium symmetry.

\section{Acknowledgments}

This research was supported by the Ministry of Education and Science of the Republic of Kazakhstan.

\section{References}

1. Tleukenov, S.K. (2014). A method for the analytical description of coupled-field waves in various anisotropic media, Acta Mechanica 225, 3535-3547.

2. Tleukenov, S.K. (2014). Method of matricant. Propagation of waves in anisotropic media, LAP Lambert Academic Publishing.

3. Tleukenov, S.K. (2014). Wave process and method of matricant, Herald of L.N.Gumilyov Eurasian National University 4, 68-74. 